\title{
Refined-mapping of the novel TSG within the 17q24.3 chromosomal region in non-small cell lung cancer samples
}

\author{
WAYREN HUANG ${ }^{1}$, YICHING WANG ${ }^{2 *}$, WOEICHYN CHU ${ }^{1 *}$ and RUOCHIA TSENG ${ }^{3}$ \\ ${ }^{1}$ Department of Biomedical Engineering, School of Biomedical Science and Engineering, National Yang-Ming University, \\ Taipei 11221; ${ }^{2}$ Department of Pharmacology, College of Medicine, National Cheng Kung University, Tainan 70101; \\ ${ }^{3}$ Department of Molecular Biology and Human Genetics, College of Life Science, Tzu Chi University, \\ Hualien 97004, Taiwan, R.O.C.
}

Received October 7, 2014; Accepted July 7, 2015

DOI: $10.3892 / 01.2016 .4812$

\begin{abstract}
Lung cancer is a leading cause of cancer mortality in Taiwan. It has been previously demonstrated that alterations to tumor suppressor genes (TSGs) are involved in the multi-step carcinogenesis process that results in the development of human cancer, including lung cancer. Both copies of the TSG must be inactivated for their function to be lost. As a result, it is important to search for the genomic regions that potentially contain TSGs and to investigate the etiological association of lung cancer with the allelic deletion of various candidate TSGs. Previous genome-wide loss of heterozygosity (LOH) data has demonstrated that the chromosome region at $17 \mathrm{q} 24.3$ was a novel and frequent $\mathrm{LOH}$ region that was associated with non-small-cell lung cancer (NSCLC). In the present study, refined mapping using 9 additional microsatellite markers was performed targeting chromosome $17 q 24.3$ by polymerase chain reaction (PCR)-LOH analysis. The allelic loss pattern across 48 available tumors indicates that the minimal deletion region was located between markers D17S1882 and D17S2193 and spanned a distance of approximately $2.7 \mathrm{Mb}$, reaching $65 \% \mathrm{LOH}$ at locus D17S1816. A putative gene, LOC51321 (AMZ2), was postulated to be the deletion target on $17 \mathrm{q} 24.3$ based on the findings from these NSCLC samples. Reverse transcription-PCR (RT-PCR) expression analysis indicated reduced expression of LOC51321 in 54\% (7/13) of the NSCLC cell lines tested and 36\% (19/53) of the NSCLC tumor tissue samples analyzed. In CL1-5-F4 cells, low mRNA and protein expression of LOC51321 were associated with the promoter hypermethylation, as determined by RT-PCR, western blotting and methylation-specific PCR assays. In addition, treatment
\end{abstract}

Correspondence to: Dr Ruochia Tseng, Department of Molecular Biology and Human Genetics, College of Life Science, Tzu Chi University, 701 Zhongyang Road, Hualien 97004, Taiwan, R.O.C.

E-mail: rctseng8@mail.tcu.edu.tw

*Contributed equally

Key words: deletion, marker, chromosome, DNA methylation, non-small cell lung cancer with 5-Aza-deoxycytosine successfully restored mRNA expression by de-methylating the putative promoter region in CL1-5-F4 cells that lacked LOC51321 expression, but did harbor the relevant methylated promoter. These findings indicate that LOC51321 may be involved in lung tumorigenesis.

\section{Introduction}

Cancer develops through a multistep process that involves mutations in certain genes; these include the inactivation of recessive tumor suppressor genes (TSGs), the activation of dominant oncogenes, and the inactivation of genes involved in DNA repair and/or replication (1). Both copies of TSG alleles have to be inactivated for their function to be lost (2). While one allele may be inactivated by point mutation, changes in methylation or a small deletion, the other allele is frequently inactivated by a large deletion that involves the whole gene of interest, as well as adjacent stretches of DNA (3). Searches for genomic regions that undergo frequent deletion in cancer samples have aided in the identification and confirmation of the location of several TSGs.

Minimal deletion region (MDR) analysis of solid tumors has enabled the delineation of specific regions that are the likely locations of critical TSGs, and have also provided a molecular portrait of the pattern of accumulation of genetic alterations during the multistep progression that leads to cancer (4-7). Confirmed TSGs may be examined by MDR analysis, since MDR consistently identifies the map positions of critical TSGs that are involved in various different types of cancer (8).

Genome-wide loss of heterozygosity ( $\mathrm{LOH})$ data has previously demonstrated that chromosome regions at $2 \mathrm{p} 23.3$, 2p24.3, 2q35, 6p22.2, 7p14.3, 7p22.2, 17q24.3 and 21q22.3 were novel and frequent $\mathrm{LOH}$ regions in non-small-cell lung cancer (NSCLC) (9). In the present study, refined mapping data obtained using 9 markers together with expression analysis, it was demonstrated that LOC51321 may be a deletion target on 17q24.3 in NSCLC tumors. LOC51321, a putative gene, named $A M Z 2$, was determined to map at $17 \mathrm{q} 24.2$ according to RefSeq. In AceView, it covers $51.26 \mathrm{~kb}$, from 63713640 to 63764901 (NCBI build 35, August 2004), on the direct strand. Putative homologs of LOC51321 have been identified in Canis familiaris, Mus musculus and Rattus norvegicus. Conserved 
domains from the CDD (conserved protein domain database) found in protein sequences by rpsblast search demonstrated that the predicted gene encoded a putative $\mathrm{Zn}$-dependent protease. Based on the above findings, it could be hypothesized that LOC51321 may potentially possess metallopeptidase activity. Therefore, the present study characterized the candidate TSG, LOC51321 (the AMZ2 gene), by analyzing its mRNA expression and promoter hypermethylation in lung cancer cell lines and samples from lung cancer patients.

\section{Materials and methods}

Sample preparation and clinical characterization of the patients. Tissues were collected after obtaining institutional review board permission from the Human Biobank of the Research Center of Clinical Medicine, Veteran General Hospital (Taipei, Taiwan) and informed consent from the recruited patients. Surgically resected tumor samples from 53 patients admitted to Veteran General Hospital with NSCLC were collected between 2002 and 2008. Of these patients, 24 had adenocarcinoma (AD), 24 had squamous cell carcinoma (SCC), and 5 had large-cell carcinoma, according to the World Health Organization classification (10). Surgically resected tumor samples were immediately snap-frozen and then subsequently stored in liquid nitrogen. Information on the gender, age, smoking history and tumor type of the patients were obtained from the Cancer Data Bank of Taipei Veteran General Hospital with de-identification of patient IDs and names performed via the Human Biobank, Research Center of Clinical Medicine, Taipei Veteran General Hospital.

For the LOH and methylation assay, genomic DNA from matched pairs of primary tumor samples and nearby normal lung tissue samples were prepared using proteinase $\mathrm{K}$ digestion and phenol-chloroform extraction, followed by ethanol precipitation (all reagents from Sigma-Aldrich, St Louis, MO, USA) (9). Serial 5- $\mu \mathrm{m}$ serial sections were cut from formalin-fixed, paraffin-embedded tumor tissues. All slides were stained with hemotoxylin and eosin (Sigma-Aldrich), and one of the slides was coverslipped and used as a guide to localize the tumor region. Tumor cells were then microdissected from up to three sections. The cells were pelleted by centrifugation at high speed $(15,115 \mathrm{x} \mathrm{g})$ for $5 \mathrm{~min}$. The DNA was extracted by digesting the cells in buffer consisting of $50 \mu \mathrm{M}$ Tris- $\mathrm{HCl}$ ( $\mathrm{pH} 8.5$ ), $1 \mu \mathrm{M}$ ethylenediamineterraacetic acid ( $\mathrm{pH} 8.0), 0.5 \%$ Tween 20 , and $200 \mathrm{mg} / \mathrm{ml}$ proteinase $\mathrm{K}$, at $55^{\circ} \mathrm{C}$ for $4-6 \mathrm{~h}$, then at $37^{\circ} \mathrm{C}$ for $24-48 \mathrm{~h}$, followed by $10 \mathrm{~min}$ of incubation at $95^{\circ} \mathrm{C}$ to destroy any remaining proteinase $\mathrm{K}$ activity. The cellular DNA was then purified using phenol/chloroform extraction followed by ethanol precipitation. Any insoluble material present in the DNA solution was pelleted by centrifugation and aliquots of supernatant were used directly in the polymerase chain reactions (PCRs). For the RNA expression assay, total RNA was prepared from tumors and normal lung tissues, using Trizol reagent (Invitrogen Life Technologies, Carlsbad, CA, USA). cDNA was then synthesized using SuperScript ${ }^{\mathrm{TM}}$ reverse transcriptase (Invitrogen Life Technologies) using the protocols provided by the manufacturer.

Minimal deletion region analysis. Genomic DNA (20 ng) from normal lung cells or from tumor cells of 48 patients were used for each PCR analysis. PCR reactions were conducted in a $10 \mathrm{ml}$ volume using $0.05 \mu \mathrm{M}$ fluorescently labeled and unlabeled primers in order to detect the microsatellite markers located in the region of interest. The microsatellite markers used were as follows: D17S1809, D17S1825, D17S1882, D17S1816, D17S807, D17S1813, D17S2193, D17S789 and D17S795. The PCR reaction contained $250 \mu \mathrm{M}$ each dNTP, $2.5 \mu \mathrm{M} \mathrm{MgCl} 2$, and 0.5 units AmpliTaq DNA polymerase (PE Applied Biosystems, Foster City, CA), and the manufacturer's instructions were followed (PE Applied Biosystems). The primer sequences were obtained from Research Genetics (Huntsville, AL, USA). Next the PCR products were mixed with fluorescent molecular weight markers and subjected to electrophoresis on a MegaBACE 1000 automatic sequencer. Allele sizes were determined using Genetic Profiler Analysis version 2.0 software. The allelic ratio was calculated as $(\mathrm{T} 1 / \mathrm{T} 2) /(\mathrm{N} 1 / \mathrm{N} 2)$, which represents the ratio of the area values for the tumor (T) alleles versus the ratio of area values for the normal $(\mathrm{N})$ alleles. $\mathrm{LOH}$ was defined as an allelic ratio $>2.0$ or $<0.5$.

Cell lines. The human lung cancer cell lines A549, H23, H226, H226Br, H1299, H1355 and H1435, and normal lung cell lines MRC5 and Beas2B were purchased from the American Type Culture Collection (Manassas, VA, USA). The human lung cancer cell lines CL1-0, CL1-1, CL1-3, CL1-5-F4, CL2 and CL3 were obtained from a Taiwanese patient with AD of the lung and was kindly provided by Dr. Pan-Chyr Yang of the Department of Internal Medicine, National Taiwan University Hospital (Taipei, Taiwan).

Analysis of LOC51321 mRNA expression using a multiplex reverse transcriptase-PCR (RT-PCR) assay. LOC51321 (AMZ2 gene) mRNA expression were assayed by multiplex RT-PCR analysis using the glyceraldehyde-3-phosphate dehydrogenase $(G A P D H)$ gene as an internal control. The coding regions of exons 3-7 of the LOC51321 (AMZ2) gene and of the $G A P D H$ gene were amplified. Sequences for the primers for the RT-PCR were as follows: LOC51321F, Forward 5'-CCAGTGCTTGTGTCACAGTATGAG-3' and LOC51321R, Reverse 5'-TCTACAATGCTGAAGCCAAC-3'. Reactions were carried out in a volume of $25 \mathrm{ml}$ with $1 \mathrm{ml}$ cDNA and 0.25 mol primers using a MyCycler ${ }^{\mathrm{TM}}$ thermal cycler (Bio-Rad Laboratories, Taipei, Taiwan). The PCR was performed for 32 cycles with an annealing temperature of $61^{\circ} \mathrm{C}$. The number of cycles, the amount of primers and the cDNA used were adjusted to provide quantitative amplification during multiplex RT-PCR. All PCR products were separated on $2 \%$ agarose gels $(100 \mathrm{~V} / 17.5 \mathrm{~cm})$ and stained with ethidium bromide. To quantify the relative levels of gene expression in the multiplex RT-PCR assay, the value for the internal standard $(G A P D H)$ in each test tube was used as the baseline value for gene expression in that sample, and a relative value was calculated for each target gene transcript amplified from each tumor and its matched normal sample. Tumor cells that exhibited mRNA expression $<50 \%$ of that of the normal cells were deemed to have an abnormal pattern of expression.

Methylation-specific PCR (MSP) assay. The methylation status in the promoter region of the LOC51321 (AMZ2) gene was determined by chemical treatment with sodium bisulfite 
Table I. Refined mapping of 17q24.3 in 48 NSCLC patients.

\begin{tabular}{lcc}
\hline Markers & STS Map (bp) & LOH\% \\
\hline D17S1809 & $64,701,223$ & 40 \\
D17S1882 & $65,916,126$ & 58 \\
D17S1816 & $66,424,320$ & 65 \\
D17S807 & $66,862,762$ & 56 \\
D17S1813 & $67,489,770$ & 52 \\
D17S2193 & $68,551,515$ & 61 \\
D17S789 & $68,632,310$ & 50 \\
D17S795 & $68,681,985$ & 19 \\
D17S1826 & $72,538,910$ & 39 \\
\hline
\end{tabular}

NSCLC, non-small cell lung cancer; STS, sequence tagged site; $\mathrm{LOH}$, loss of heterozygosity.

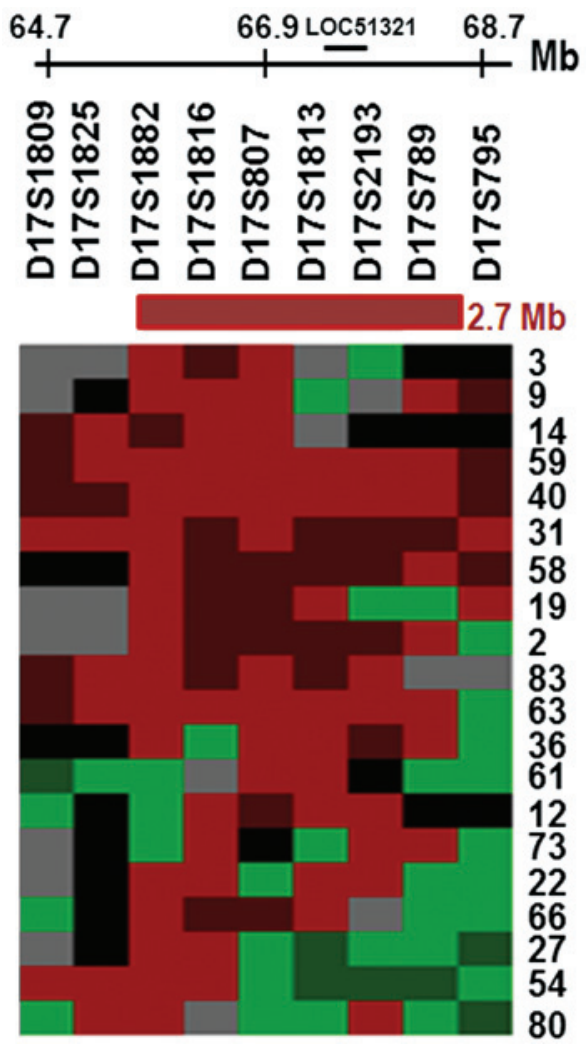

Figure 1. Schematic representation of the deleted regions at chromosomal region $17 \mathrm{q} 24.2-24.3$ in 20 representative NSCLC tumors. At the top are the nine microsatellite markers, which are listed from centromere (left) to telomere (right). Numbers on the right identify the tumors. The minimal deleted region is indicated as a horizontal bar on the top of the diagram. Color codes: red box, LOH; dark red, non-informative area bordered by two regions of LOH; green box, retention of heterozygosity; dark green box, non-informative area bordered by at least one region of retention of heterozygosity; gray box, non-informative; black box, non-determined; NSCLC, non-small cell lung cancer; LOH, loss of heterozygosity.

and subsequent MSP analysis. In total, $500 \mathrm{ng}$ of genomic DNA was denatured at $95^{\circ} \mathrm{C}$ for $5 \mathrm{~min}$ and then in the presence of $\mathrm{NaOH}$ (final concentration, $0.2 \mathrm{M}$ ) incubated at $37^{\circ} \mathrm{C}$ for $15 \mathrm{~min}$. Next, $10 \mu \mathrm{M}$ hydroquinone (Sigma-Aldrich), and $3 \mathrm{M}$ sodium bisulfite (Sigma-Aldrich) were added and the mixture was incubated at $50^{\circ} \mathrm{C}$ for $18 \mathrm{~h}$. After these treatments, the modified DNA was purified using a Microcon YM-50 DNA purification column (Millipore, Bedford, MA, USA). Treatment of the genomic DNA with sodium bisulfite converted unmethylated but not methylated cytosines to uracils, which were then converted to thymidines during the subsequent PCR step. The primers used for the converted sequence were as follows: LOC51321MF, Forward 5'-CGGAGTCGTCGCGAGCGT AA-3' and LOC51321MR, Reverse 5'-CGTCGCGCACGC CCTAT-3'; LOC51321UF, Forward 5'-GTTGTGGAGTTG TTGTGAGTGTAA-3' and LOC51321UR, Reverse 5'-ACC ACTACATCACACACACCCTA-3'. The PCR was performed for 35 cycles with annealing temperatures of $64^{\circ} \mathrm{C}$ and $50^{\circ} \mathrm{C}$ for the unmethylated and methylated reactions, respectively, using $100 \mathrm{ng}$ bisulfite-modified DNA. All PCR products were separated on $2 \%$ agarose gels and stained with ethidium bromide. Negative and positive control samples with unmethylated lymphocyte DNA and SssI methyltransferase treated methylated DNA were also included in each set of PCR amplifications. The lymphocyte DNA was obtained by collecting blood samples from a healthy volunteer Mr. Wayren Huang, one of the investigators. The lymphocyte DNA were isolated using a Genomic DNA Extraction Miniprep System (Viogene BioTek Corp., New Taipei City, Taiwan). Hypermethylation was defined as amplification of more methylated product than unmethylated product from a tumor sample.

5-Aza-2'-deoxycytidine (5-Aza-dC) treatment of lung cancer cells. The human lung cancer cell lines CL1-1 and CL1-5-F4 were obtained from a Taiwanese patient with AD of the lung and was kindly provided by Dr. P-C Yang, Department of Internal Medicine, National Taiwan University Hospital. Cells were plated at $10^{5}$ cells/100-mm culture dish on the day before treatment. The cultures were treated for 3 doubling times with $1 \mathrm{uM}$ of 5-Aza-dC. The 5-Aza-dC-containing medium was changed after each cell doubling during the treatment. On the day after the third doubling, the cells were harvested for an analysis of their methylation status using an MSP assay as described above, and for LOC51321 mRNA expression using an RT-PCR assay.

Statistical analysis. Pearson's $\chi^{2}$ test was used to compare the frequency of LOH in NSCLC patients. All data were each assigned a number and a color code as follows: the existence of LOH in a marker of NSCLC was assigned the number -1 and red color; the retention of a chromosomal region was assigned the number +1 and green color; and the noninformative data were assigned black color; no data were assigned gray color. The color-coding system provides interactive graphic analysis of clustering results in the TreeView program (http://jtreeview.sourceforge.net) (9). Based on a previously described clustering algorithm the similarity of $\mathrm{LOH}$ profiles of a group of tumor samples (such as a subgroup of NSCLC features) was calculated (9). $\mathrm{P}<0.05$ was considered to indicate a statistically significant difference. SPSS software, version 19.0 (IBM, Chicago, IL, USA) was used for all statistical analyses.

\section{Results}

Refined mapping of the $\mathrm{LOH}$ region on $17 q 24.3$ and expressional validation of LOC51321 using NSCLC cell lines and 
Table II. Association between LOH and patient clinicopathological parameters ${ }^{\mathrm{a}}$.

\begin{tabular}{lllllll}
\hline Variable & Markers & Categories & No LOH & LOH
\end{tabular}

\section{Smoking}

Marker associated with smoker patients

$\begin{array}{lcccc}\text { D17S1882 } & \text { No } & 48 \% & 52 \% & 0.888 \\ & \text { Yes } & 45 \% & 55 \% & \\ \text { D17S1816 } & \text { No } & 29 \% & 71 \% & 0.658 \\ & \text { Yes } & 38 \% & 62 \% & \\ \text { D17S807 } & \text { No } & 75 \% & 25 \% & \mathbf{0 . 0 3 2} \\ & \text { Yes } & 29 \% & 71 \% & \\ \text { D17S1813 } & \text { No } & 35 \% & 65 \% & 0.183 \\ & \text { Yes } & 67 \% & 33 \% & \\ \text { D17S2193 } & \text { No } & 40 \% & 60 \% & 0.907 \\ & \text { Yes } & 38 \% & 62 \% & \\ \text { D17S789 } & \text { No } & 75 \% & 25 \% & \mathbf{0 . 0 2 2} \\ & \text { Yes } & 31 \% & 69 \% & \end{array}$

\section{Tumor type}

Marker associated with tumor type

$\begin{array}{lcccc}\text { D17S1882 } & \text { AD } & 52 \% & 48 \% & 0.463 \\ & \text { SCC } & 40 \% & 60 \% & \\ \text { D17S1816 } & \text { AD } & 22 \% & 78 \% & 0.279 \\ & \text { SCC } & 45 \% & 55 \% & \\ \text { D17S807 } & \text { AD } & 80 \% & 20 \% & \mathbb{0 . 0 0 3} \\ & \text { SCC } & 20 \% & 80 \% & \\ \text { D17S1813 } & \text { AD } & 44 \% & 56 \% & 0.305 \\ & \text { SCC } & 43 \% & 57 \% & \\ \text { D17S2193 } & \text { AD } & 29 \% & 71 \% & 0.940 \\ & \text { SCC } & 44 \% & 56 \% & \\ \text { D17S789 } & \text { AD } & 42 \% & 58 \% & 0.445 \\ & \text { SCC } & 56 \% & 44 \% & \\ & & & & \end{array}$

Large-cell carcinoma patients are not included due to the small sample size $(n=5)$. LOH, loss of heterozygosity; AD, adenocarcinoma; SCC, squamous cell carcinoma.

A

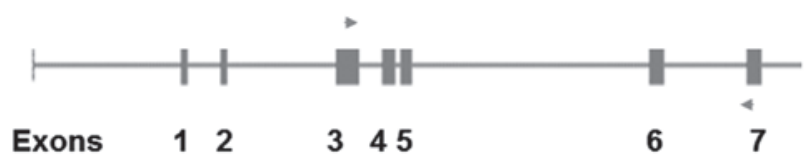

B

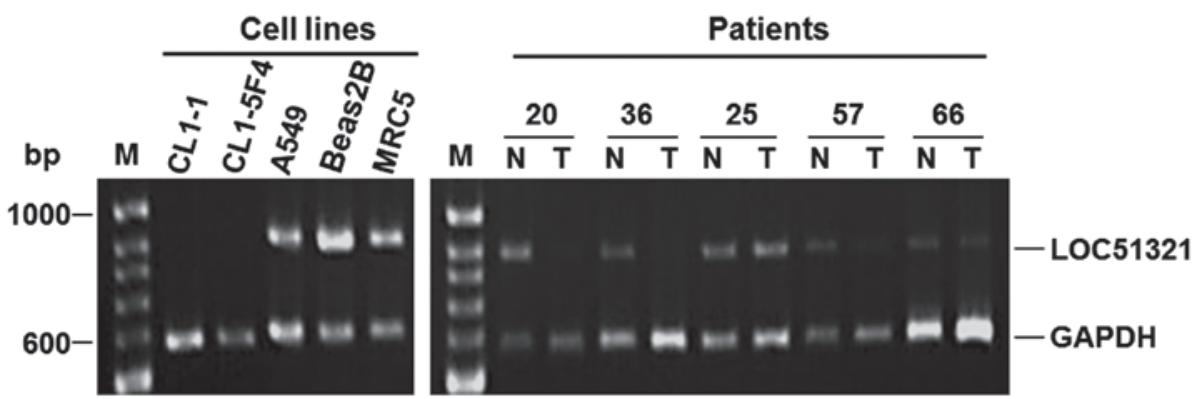

Figure 2. Expression level of LOC1321 in lung cancer cell lines and lung cancer patients. (A) Schematic structure of LOC51321 gene. The exons are shown as squares and the positions of primers used are given as arrows. (B) Representative figures for mRNA expression analysis of the LOC51321 gene. Multiplex PCRs were performed by mixing LOC51321 primers and internal control primers for the GAPDH gene. NSCLC cell lines CL1-1, CL1-5F4 and A549 and patient 57 were negative for LOC51321 mRNA expression. Normal lung cell lines Bes6 and MRC5 were positive for LOC51321 mRNA expression. PCR, polymerase chain reaction; NSCLC, non small cell lung cancer. 
A

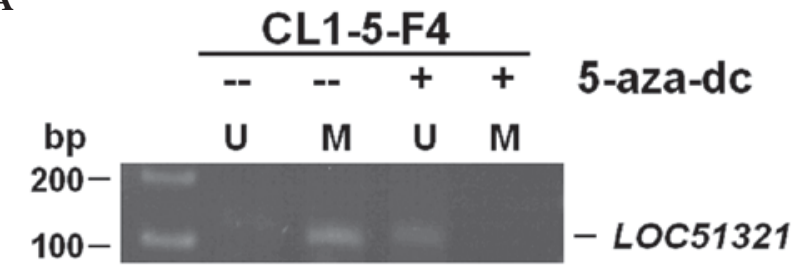

B

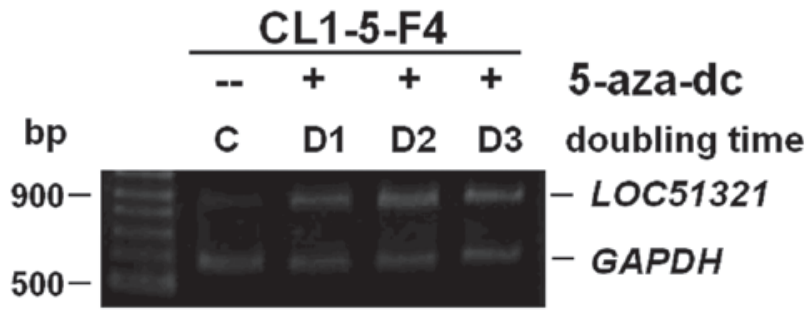

Figure 3. Effects of 5-Aza-dC treatment on CL1-5-F4 cells. (A) Demethylation analysis of LOC51321 in lung cancer cell lines after 5-Aza-dC treatment using the MSP assay. The presence of the Uracil product after 5-Aza-dC treatment indicates the presence of demethylation of the promoter in these cell lines. (B) RT-PCR analysis of the mRNA expression of the LOC51321 in cells. RT-PCR, reverse transcription-polymerase chain reaction; MSP, methylation specific PCR.

patient samples. Since the chromosome region at $17 \mathrm{q} 24.3$ has not previously been identified as a high frequency loss region in NSCLC samples, the present study further refined the LOH pattern of the chromosomal region 17q24.2-24.3 using 9 additional microsatellite markers. This was conducted using a panel of 48 microdissected NSCLC tumor samples and their matched normal lung tissue samples. The allelic loss pattern of these tumors indicates that the MDR was located between markers D17S1882 and D17S789, reaching 50-65\% $\mathrm{LOH}$ at this locus (Table I). A total of 32/42 (79\%) informative tumors exhibited $\mathrm{LOH}$ that was associated with this region. Markers D17S807 and D17S789 were specifically associated with patients who smoked $(\mathrm{P}=0.03$ and 0.02 , respectively, Table II). In addition, marker D17S807 was also demonstrated to be related to SCCs ( $\mathrm{P}=0.003$, Table II). Fig. 1 summarizes the $\mathrm{LOH}$ results for the 20 representative tumors that exhibited allelic imbalance, which spanned a distance of approximately $2.7 \mathrm{Mb}$. The smallest commonly deleted region included markers that are located within LOC51321, a putative AMZ2 gene that encodes a protein with a predicted $\mathrm{Zn}$-dependent protease domain. Therefore, alterations within LOC51321 may occur in NSCLC cell lines and/or tumor samples.

The alteration analysis of LOC1321 in lung cancer. The expression level of $L O C 1321$ was then analyzed in two normal lung cancer lines, 13 NSCLC cell lines, and 53 matched normal and tumor tissues by semiquantitative multiplex RT-PCR. This analysis demonstrated that there was reduced expression of LOC51321 in 54\% (7/13) of the NSCLC cell lines and 36\% (19/53) of the tumor tissues (Fig. 2).

Re-activation of LOC51321 by 5-Aza-dC treatment. Aberrations in promoter methylation are considered to be important in the development of lung cancer (11). To determine whether epigenetic change altered gene expression, promoter hypermethylation of the LOC51321 was assessed using MSP analysis in lung cancer cells. One lung cancer cell line CL1-5F4 that demonstrated negative expression together with promoter hypermethylation of the LOC51321 gene was treated with the demethylating agent 5-Aza-dC (Fig. 3A). As presented in Fig. 3B, 5-Aza-dC successfully restored mRNA and protein expression by de-methylating the putative promoter region in the cells that lacked LOC51321 expression and also harbored an appropriately methylated promoter.

\section{Discussion}

Both copies of TSGs need to be inactivated for their function to be lost (2). One allele may be inactivated by a point mutation, a methylation change, or a small deletion. Generally, the other allele is often inactivated by a large deletion that involves the gene of interest as well as adjacent stretches of DNA (3). Thus, searches for genomic regions frequently deleted in cancer have aided in the identification and/or confirmation of the location of several TSGs. To the best of our knowledge, the present study reports, for the first time, a high frequency loss region on chromosomes 17q24.3 in NSCLC samples. Based on refined mapping using 9 markers together with an expressional study involving semiquantative RT-PCR, the present study identified a novel gene LOC51321 that may be one of the main deletion targets on $17 \mathrm{q} 24.3$ in NSCLC. LOC51321 was demonstrated to exhibit loss of mRNA expression in 47 and $36 \%$ of examined NSCLC cell lines and tumor samples, respectively. According to the annotated mRNA database, LOC51321 may be able to produce a series of transcripts by alternative splicing. The primers for RT-PCR in the present study were deliberately designed to cover a large region and to target sequences not likely to be involved in alternative splicing. Therefore, if any alternative transcripts occurred, these should have been detected. However, no aberrant sized cDNA product was produced by any lung cancer sample, which indicates that LOC51321 may be a simple gene with a single open reading frame product. Nevertheless, probable alternatives in terms of promoters and the possibility of alternative splicing outside the region examined need to be investigated in the future.

Alterations in mRNA expression associated with promoter hypermethylation of the LOC51321 gene in CL1-5-F4 cell line were explored and the findings indicate that promoter hypermethylation may be one possible source of inactivation of the LOC51321 gene. This conclusion is further strengthened by the additional findings of the present study that the re-expression of the mRNA expression together with de-methylation at the promoter region in the LOC51321 genes using 5-Aza-dC treatment of a lung cancer cell line.

Based on annotation information from www.genecards.org, LOC31521 (AMZ2) is predicted to be a $\mathrm{Zn}$-dependent protease. The $\mathrm{Zn}$-dependent and calcium-dependent family of proteins termed the matrix metalloproteinases have been demonstrated to be collectively responsible for the degradation of the extracellular matrix (12). Members of this family, including metalloendopeptidase, collagenases, stromelysins and gelatinases; 
and are involved in routine tissue remodelling processes, such as wound healing, embryonic growth and angiogenesis (12). Loss of expression of some metalloendopeptidases has been demonstrated to be involved in tumorigenesis. Shipp et al (13) demonstrated that malignant pulmonary neuroendocrine cells expressed low levels of the cell surface metalloendopeptidase CD10/neutral endopeptidase 24.11 (CD10/NEP, common acute lymphoblastic leukemia antigen) and that this enzyme hydrolyzes bombesin-like peptides (13). The growth of bombesin-like peptide-dependent cancer cells is inhibited by CD10/NEP. The results provide evidence that $\mathrm{CD} 10 / \mathrm{NEP}$ is involved in the regulation of tumor cell proliferation. Since cigarette smoke inactivates CD10/NEP (13), reduced cell surface CD10/NEP enzymatic activity may be causally associated with the development of lung cancer.

Further characterization, including identifying the various mutations involved in gene inactivation, performing promoter identification, and conducting a full analysis of protein(s) associated with LOC51321 will be instrumental in confirming the mRNA expression findings presented in the current study. An understanding of the structure, nature, and function of this chromosomal location will also be necessary to determine its full role in carcinogenesis.

\section{Acknowledgements}

The authors would like to express their thanks to Dr. Han Shui Hsu (Chief in Division of Thoracic Surgery, Taipei Veterans General Hospital, Taipei, Taiwan) for providing the clinical samples. The present study was supported in part by the Ministry of Science and Technology (No. 101-2320-B-320-008).

\section{References}

1. Fearon ER: Human cancer syndromes: Clues to the origin and nature of cancer. Science 278: 1043-1050, 1997.

2. Knudson AG: Hereditary cancer: Two hits revisited. J Cancer Res Clin Oncol 122: 135-140, 1996.

3. Kohno T and Yokota J: How many tumor suppressor genes are involved in human lung carcinogenesis? Carcinogenesis 20: 1403-1410, 1999.

4. Vogelstein B, Fearon ER, Hamilton SR, Kern SE, Preisinger AC, Leppert M, Nakamura Y, White R, Smits AM and Bos JL: Genetic alterations during colorectal-tumor development. N Engl J Med 319: 525-532, 1988.

5. Lasko D, Cavenee W and Nordenskjöld M: Loss of constitutional heterozygosity in human cancer. Annu Rev Genet 25: 281-314, 1991.

6. Yokota J and Sugimura T: Multiple steps in carcinogenesis involving alterations of multiple tumor suppressor genes. FASEB J 7: 920-925, 1993.

7. Gray JW and Collins C: Genome changes and gene expression in human solid tumors. Carcinogenesis 21: 443-452, 2000.

8. Thiagalingam S, Foy RL, Cheng KH, Lee HJ, Thiagalingam A and Ponte JF: Loss of heterozygosity as a predictor to map tumor suppressor genes in cancer: Molecular basis of its occurrence. Curr Opin Oncol 14: 65-72, 2002.

9. Tseng RC, Chang JW, Hsien FJ, Chang YH, Hsiao CF, Chen JT, Chen CY, Jou YS and Wang YC: Genomewide loss of heterozygosity and its clinical associations in non small cell lung cancer. Int J Cancer 117: 241-247, 2005.

10. Brambilla E, Travis WD, Colby TV, Corrin B and Shimosato Y: The new World Health Organization classification of lung tumours. Eur Respir J 18: 1059-1068, 2001.

11. Zhao Y, Zhou H, Ma K, Sun J, Feng X, Geng J, Gu J, Wang W, Zhang $\mathrm{H}, \mathrm{He} \mathrm{Y}$, et al: Abnormal methylation of seven genes and their associations with clinical characteristics in early stage non-small cell lung cancer. Oncol Lett 5: 1211-1218, 2013.

12. Borkakoti N: Structural studies of matrix metalloproteinases. J Mol Med (Berl) 78: 261-268, 2000.

13. Shipp MA, Tarr GE, Chen CY, Switzer SN, Hersh LB, Stein H, Sunday ME and Reinherz EL: CD10/neutral endopeptidase 24.11 hydrolyzes bombesin-like peptides and regulates the growth of small cell carcinomas of the lung. Proc Natl Acad Sci USA 88: 10662-10666, 1991. 